\title{
Static Aerodynamics of the Mars Exploration Rover Entry Capsule
}

\author{
Mark Schoenenberger, F. McNeil Cheatwood ${ }^{\dagger}$ \\ and Prasun N. Desai ${ }^{\ddagger}$ \\ NASA Langley Research Center, Hampton, Virginia 23681
}

\begin{abstract}
The static aerodynamics for the Mars Exploration Rover (MER) aeroshell are presented. This aerodynamic database was an integral part of the end-to-end simulation used in preentry analysis for determining the MER entry design requirements for development of the MER entry system, as well as targeting the MER landing sites. The database was constructed using the same approach used for Mars Pathfinder (MPF). However, the MER aerodynamic database is of much higher fidelity and tailored to the MER entry trajectories. This set of data includes direct simulation Monte Carlo calculations covering the transitional regime of the entry trajectory and computational fluid dynamics calculations describing the aerodynamics in the hypersonic and supersonic continuum regimes. An overview of the methodology used to generate the data is given along with comparisons to important features in the MPF aerodynamics and related heritage data. The MER and MPF comparison indicates that trajectory specific data is required to properly model the flight characteristics of a blunt entry capsule at Mars.
\end{abstract}

\section{Nomenclature}

$\begin{array}{llll}C_{A} & \text { Axial force coefficient } & n & \text { Number density, particle } / m^{3} \\ C_{l} & \text { Roll moment coefficient } & S & \text { Reference area, } m^{2} \\ C_{m} & \text { Pitch moment coefficient } & V & \text { Freestream velocity, } m / s \\ C_{m_{q}} & \text { Pitch damping coefficient } & x, y, z & \text { Cartesian position dimensions } \\ C_{n} & \text { Yaw moment coefficient } & \alpha & \text { Angle-of-attack, deg } \\ C_{N} & \text { Normal force coefficient } & \beta & \text { Angle-of-sideslip, deg } \\ C_{p} & \text { Pressure coefficient } & \lambda & \text { Mean free path } \\ C_{Y} & \text { Side force coefficient } & \text { Subscripts } & \\ \bar{d} & \text { Molecular diameter, } m & h s & \text { Hard sphere } \\ D & \text { Reference diameter, } m & T & \text { Total } \\ K n & \text { Knudsen number } & c g & \text { Center-of-gravity } \\ M & \text { Mach number } & b & \text { Base }\end{array}$

\section{Introduction}

The Mars Exploration Rovers (MER) "Spirit" and "Opportunity" successfully landed on Mars on January 3rd and 24th, 2004. Entering the Mars atmosphere at $5500 \mathrm{~m} / \mathrm{s}$, each rover was slowed to just over $400 \mathrm{~m} / \mathrm{s}$ in four minutes by a blunt sphere-cone heatshield. The passive ballistic entry of the MER capsules relied solely on aerodynamic stability to traverse all of the flight regimes. Therefore, accurate characterization of

*Aerospace Engineer, Exploration Systems Engineering Branch, Member AIAA

${ }^{\dagger}$ Aerospace Engineer, Exploration Systems and Space Operations Technology Directorate, Senior Member AIAA

${ }^{\ddagger}$ Aerospace Engineer, Exploration Systems Engineering Branch, Associate Fellow AIAA 
the aerodynamics was required to determine the design requirements of the MER entry system to ensure that the capsule flew properly through the atmosphere (i.e. small angles-of-attack). In addition, accurate aerodynamics were necessary to target the desired landing sites.

This paper will detail aerodynamics of the MER capsule in the flight regimes from entry interface down to parachute deployment conditions. The different flight regimes are defined by the dominant flow physics along a particular portion of the entry trajectory. The regimes, starting from atmospheric interface include: free molecular (where the particles of the atmosphere can be modeled separately without interaction with each other), transitional (where collisions among atmosphere molecules are important, but the flow cannot


tions, with possible nonequilibrium gas chemistry effects, and negligible base pressure contributions to the aerodynamics) and supersonic continuum (where flow chemistry is in equilibrium and the backshell base pressure distribution makes a significant contribution to the vehicle aerodynamics).

The MER capsule geometry analyzed for the prefiight database and the coordinate system used is shown in Figure 1. The outer mold line (OML) consists of a $70^{\circ}$ sphere-cone heatshield and a $43^{\circ}$ truncated conic backshell.

The geometry in Figure 1 is essentially the same geometry as the Mars Pathfinder entry capsule. To accommodate the addition of rocket motors late in the MER development process, three small hemispherical covers were added to the backshell (roughly halfway aft of the shoulder, $120^{\circ}$ apart) to protect the motors from wake flow. The region around the back plane of the backshell was also enlarged slightly to add volume for the parachute. Subsequent computational analyses indicated that these modifications did not significantly alter the aerodynamics of the capsule. The analysis reported in this paper does not include these modifications.
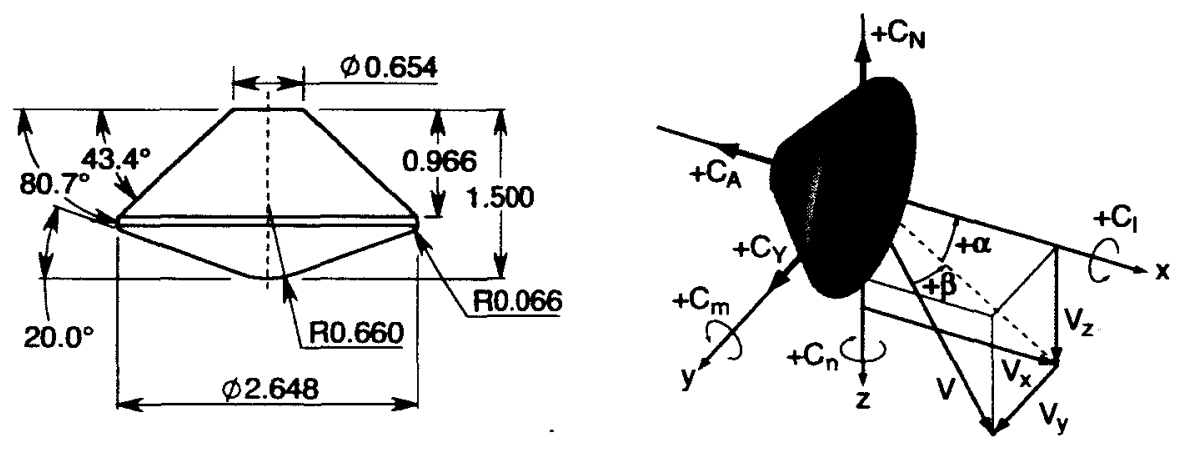

Figure 1. Entry capsule dimensions (meters) and coordinate system.

The entry trajectory for which the MER data points were calculated is shown in Figure 2. The velocity in the atmosphere peaks at roughly $5570 \mathrm{~m} / \mathrm{s}$, which is between the entry velocities of the Viking landers and Mars Pathfinder. Therefore, the analysis performed to obtain the MER database has two sets of flight data (with inherent computational and experimental heritage) by which the flight envelope is bounded. The solid line represents preflight predictions of the velocity history for the two rovers. Data points were calculated along this trajectory using the DSMC Analysis Code (DAC) ${ }^{1}$ for the transitional regime, and the Langley Aerothermodynamic Upwind Relaxation Algorithm (LAURA) ${ }^{2}$ CFD code for the continuum regime. The points indicate the conditions at which the database was calculated. Note that the higher velocity LAURA solutions were calculated for an early reference trajectory, but were sufficiently close to accurately describe the aerodynamics for the final trajectories.

\section{Methods}

The final form of the MER aerodynamic database is a FORTRAN subroutine that is called within the trajectory simulations of the entry, descent and landing (EDL) sequence. These simulations are run in the Program to Optimize Simulated Trajectories II (POST II). ${ }^{3}$ At every time step in the simulation, the variables required to characterize state of the vehicle (velocity, Knudsen number, Mach number, angle-of-attack and 


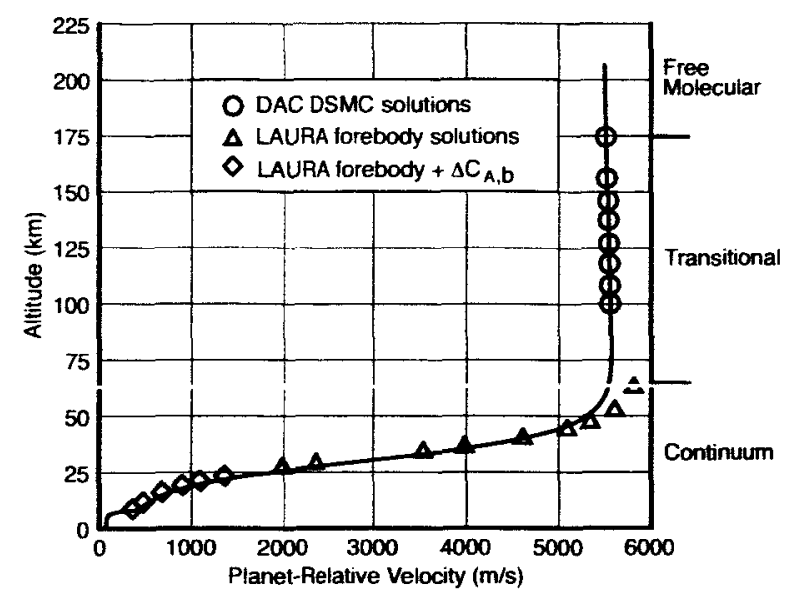

Figure 2. MER entry trajectory

angle-of-sideslip) are passed to the aerodatabase subroutine. The state variables are then used to look up the appropriate aerodynamic coefficients and return them to POST so that the forces and moments acting on the vehicle can be integrated and thus determine the motion of the vehicle through Mars' atmosphere. The range of data points was selected to encompass all possible angle-of-attack excursions that could potentially occur in Monte Carlo dispersion analysis. Different analyses and experimental work were used to determine the static and dynamic aerodynamics for each regime. Each approach was chosen in an attempt to address the relevant flow physics in a particular regime.

A schematic of the database is shown in Figure 3. This graphic gives an overview of the data sources and the boundaries which define the sets of data calculated in the various flight regimes. The static aerodynamic data is linearly bridged when switching from freestream velocity to Mach number as the independent variable, as well as when switching from forebody-only solutions to forebody solutions with a Viking derived base pressure correction. For the dynamic stability terms, the ballistic range derived data are similarly bridged to analytic (Newtonian) solutions ${ }^{4}$ of a sharp $70^{\circ}$ cone. The methods used in each regime are detailed here.

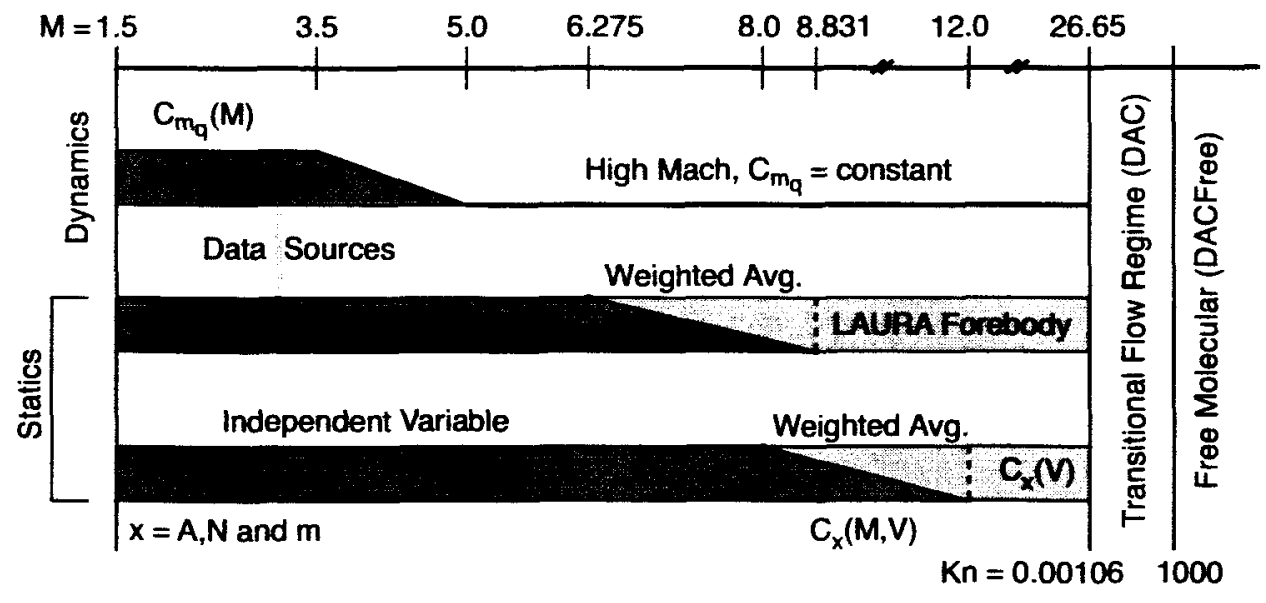

Figure 3. Schematic of MER aerodynamic database 


\section{A. Transitional and Free Molecular Calculations}

In the transitional and free molecular regimes, the fluid densities are so small that the flow can not be modeled as a continuum. In free molecular flow, even collisions between individual molecules occur so infrequently that they need not be modeled. The direct simulation Monte Carlo (DSMC) method ${ }^{5}$ is commonly used to characterize the flow in these regimes. For the transitional flow regime, static aerodynamics data were calculated with the DAC DSMC code. DAC uses a two level Cartesian grid. The first level is a uniform structured rectangular mesh and the second level is a locally refined mesh within each first level cell. Molecular collisions are calculated using the variable hard sphere model developed by Bird and wall collisions were assumed to be fully diffuse, a conservative assumption. In the transitional regime, the dependent variable against which the aerodynamic data is indexed is the hard sphere Knudsen number:

$$
K n_{h s}=\frac{\lambda}{D}=\frac{1}{\sqrt{2} \pi \bar{d}^{2} n D}
$$

Here, $\lambda$ is the mean free path of the of the local freestream gas, $\mathrm{D}$ is the base diameter of the capsule, $\bar{d}$ is the hard sphere diameter of a carbon dioxide molecule $\left(4.64 E^{-10} \mathrm{~m}\right)$ and $\mathrm{n}$ is the number density of molecules in the freestream.

Free molecular aerodynamic data, where density is so low that collisions between molecules can be ignored, were calculated with the DACFREE code. The DSMC portion of this code is essentially identical to the formulations used in DAC although collisions are not modeled. Free molecular data was calculated at $2^{\circ}$ increments from $0^{\circ}$ to $180^{\circ}$ at a Knudsen number of 1000 .

The Mars atmosphere was modeled in two ways. At higher Knudsen numbers, the atmosphere was assumed to be completely $\mathrm{CO}_{2}$, while at lower Knudsen numbers, where chemical reactions can become important, the atmosphere was modeled with mole fractions of $97.3 \% \mathrm{CO}_{2}$ and $2.7 \% \mathrm{~N}_{2}$ respectively. Table 1 lists the data points calculated in the transitional regime. For Knudsen number points where both $\mathrm{CO}_{2}$ and $\mathrm{CO}_{2} / \mathrm{N}_{2}$ solutions were generated ( $\mathrm{Kn}=3$ and 10$)$, cases were run to verify that the gas model did not alter the aerodynamic coefficients. For each point, the gas model used and the approximate minimum $\lambda / d s$ across the entire grid, is listed. The $\lambda / d s$ parameter is the number of local mean-free-paths per local cell length. Common DSMC practice says that roughly 3 mean-free-paths per cell are required for the DSMC method to accurately simulate a flow. Typically, however, $\lambda / d s$ values of 1 or smaller at the highest density points in the flow (the stagnation region) do not adversely effect the calculation of aerodynamic coefficients. Grid sensitivity studies of these MER cases show that the grids used for the data presented here were sufficiently resolved for aerodynamic forces and moments.

All data points were calculated at a velocity of $5571 \mathrm{~m} / \mathrm{s}$ as POST trajectory analyses showed that there is no significant change in velocity in the transitional regime. The wall temperature for each case was held fixed at $300 \mathrm{~K}$. DAC has the limitation that only one wall temperature may be prescribed over the geometry. Rather than attempt to predict a weighted average wall temperature based on rarefied heating calculations, it was decided to keep the wall temperature fixed at a fairly cold value and quantify the uncertainties that may be introduced by an incorrect wall temperature.

Table 1. Transitional Flight Regime Data Points (Gas model / $\frac{\lambda}{d s}$ )

\begin{tabular}{cccccccccccc}
$K n_{h s}$ & \multicolumn{2}{c}{$\alpha=0^{\circ}$} & \multicolumn{2}{c}{$6^{\circ}$} & \multicolumn{2}{c}{$10^{\circ}$} & \multicolumn{2}{c}{$16^{\circ}$} & \multicolumn{2}{c}{$20^{\circ}$} \\
\hline \hline & Gas & $\lambda / d s$ & Gas & $\lambda / d s$ & Gas & $\lambda / d s$ & Gas & $\lambda / d s$ & Gas & $\lambda / d s$ \\
\hline \hline 100 & $C O_{2}$ & 65 & $C O_{2}$ & 43 & $C O_{2}$ & 64 & $C O_{2}$ & 59 & $C O_{2}$ & 69 \\
30 & Mars & 19 & $C O_{2}$ & 17 & $C O_{2}$ & 18.5 & $C O_{2}$ & 17 & $C O_{2}$ & 17.3 \\
10 & Mars & 5.8 & $C O_{2}$ & 13.2 & Mars & 13.8 & $C O_{2}$ & 5.3 & $C O_{2}$ & 13 \\
3 & Mars & 1.4 & $C O_{2}$ & 1.3 & Mars & 1.4 & $C O_{2}$ & 1.3 & $C O_{2}$ & 1.3 \\
1 & Mars & 2.8 & Mars & 1.3 & Mars & 1.0 & Mars & 0.4 & Mars & 1.0 \\
0.30 & Mars & 0.9 & Mars & 0.9 & Mars & 0.8 & Mars & 0.5 & Mars & 0.5 \\
0.10 & Mars & 0.8 & Mars & 0.5 & Mars & 0.3 & Mars & 0.08 & Mars & 0.3 \\
\hline
\end{tabular}




\section{B. Continuum CFD Calculations}

Computational fluid dynamics (CFD) calculations were used to generate the entire set of data in the continuum regime for the MER aerodynamic database. Several other sources of static aerodynamics for $70^{\circ}$ sphere-cones were compared to the MER database to validate the data and guide the methodology used to generate the flight database. The reference data included Viking wind tunnel ${ }^{6}$ and ballistic range data, ${ }^{7}$ MER ballistic range static data ${ }^{8}$ and the Mars Pathfinder database. For the MER calculations, laminar, nonblowing, thin-layer Navier-Stokes solutions were generated with the LAURA CFD code. ${ }^{2}$ LAURA is a finite volume, shock capturing algorithm capable of calculating flows in chemical and thermal non-equilibrium. The solutions presented herein are for the thin-layer Navier-Stokes equations. In all cases, surface wall temperatures were computed using the radiative equilibrium wall temperature assumption with an emissivity of 0.9. This emissivity is that of charred SLA-561, the MER heatshield material. ${ }^{9}$ The calculations were performed on SGI Origin2000 computers (32-bit word length) with LAURA version 4.9.1. The calculations for a given solution were spread over multiple processors via Message Passing Interface (MPI). ${ }^{10}$ The matrix of CFD solutions generated for the MER database is given in Figure 4. This figure was taken from an overview paper of the MER entry trajectory analysis by Desai et al. ${ }^{11}$ The hypersonic solutions and supersonic solutions are indicated as well as the velocity, Mach number and angle-of-attack for each point.

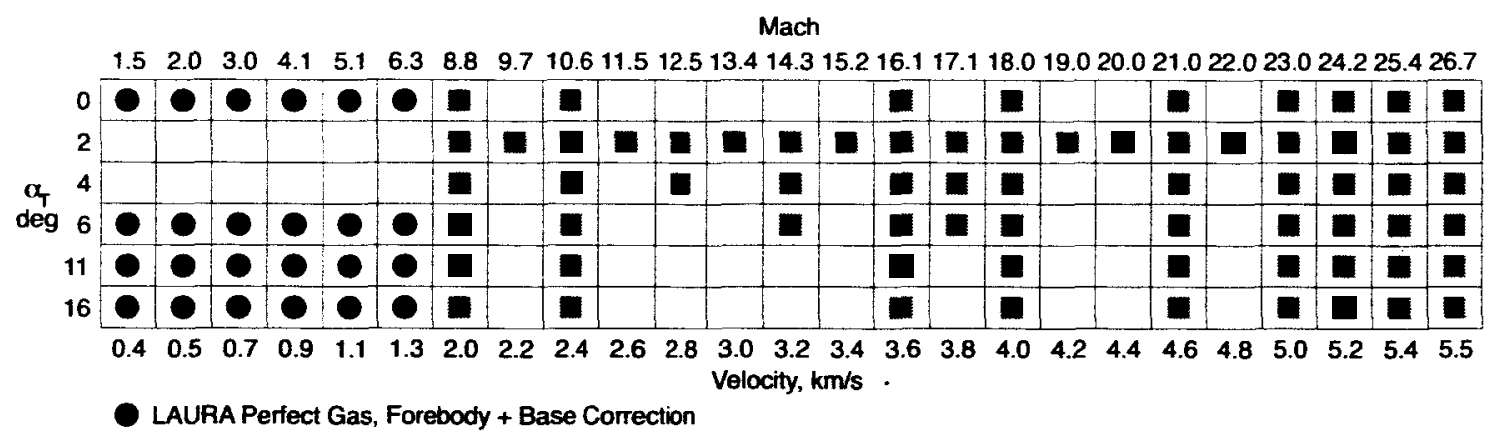

LAURA Nonequilibrium, Forebody Only

Figure 4. Matrix of LAURA solutions used in MER aerodynamic database

\section{Hypersonic Static Calculations}

The hypersonic regime begins when the flow over the forebody of the capsule becomes dense enough to be modeled as a continuum. For this database, this transition occurs at a freestream Knudsen number of 0.001 . In hypersonic continuum, the static aerodynamics is almost entirely due to the forebody pressure distribution. The aftbody and shear contributions are negligible.

For the hypersonic portion of the trajectory, the flow was assumed to be in chemical (8-species, no ionization) and thermal (separate translational and vibrational temperatures) non-equilibrium. For the surface catalysis, mass fractions were set to their values at the freestream temperature. The hypersonic grid is a structured, 7-block forebody-only grid. The block containing the capsule nose is dimensioned $11 \times 11$ at the wall and extends 64 cells into the flow. The remaining 6 blocks which extend back to the capsule shoulder ( 2 blocks in the streamwise direction, 3 blocks circumferentially) are dimensioned $13 \times 11$ along the wall (with 13 cells in the streamwise direction along the cone) and extend 64 cells into the flow as well. Figure 5 shows a sample grid used for these solutions.

\section{Supersonic Static Calculations}

LAURA was used again for the supersonic flow regime. For these solutions a pole-boundary (axisymmetric) half-model grid was used. A singularity free grid can be important for aeroheating solutions but was not necessary for aerodynamic forces. The grid has two blocks on the forebody and 3 on the backshell. The grid extended 64 cells into the flow as was done for the hypersonic solutions. Extensive grid resolution 


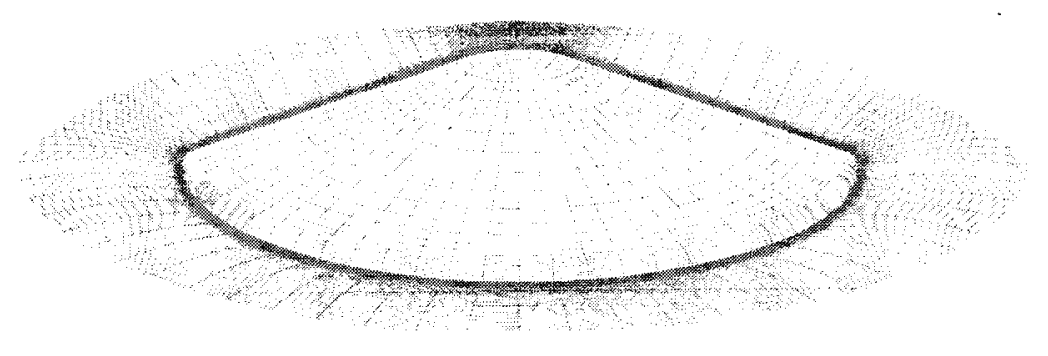

Figure 5. Example hypersonic grid

studies were performed and showed that the hypersonic and supersonic grids are sufficiently resolved. For the supersonic portion of the trajectory, the flow was treated as a perfect gas using an effective freestream ratio of specific heats $(\gamma)$ appropriate for the local flow conditions.

Although a solution over the entire capsule was generated in each case, comparisons with heritage data suggested that LAURA was incorrectly calculating the base contribution to $C_{A}$ at low Mach numbers (below $\mathrm{M}=2.5$ ). As a result, the approach used for Mars Pathfinder was adopted. The forebody contribution was taken from the full solutions. A base correction was used for the contribution to $C_{A}$ due to the backshell pressure distribution. The base pressure correction, determined in preparation for the MPF mission by fitting a curve through Viking flight data, is described by the following relation:

$$
\Delta C_{A, b}=C_{p, b}=a_{o}+\frac{a_{1}}{M_{\infty}}+\frac{a_{2}}{M_{\infty}^{2}}+\frac{a_{3}}{M_{\infty}^{3}}
$$

Where

$$
\begin{aligned}
& a_{0}=8.325 E^{-03} \\
& a_{1}=1.129 E^{-01} \\
& a_{2}=-1.801 E^{-00} \\
& a_{3}=1.288 E^{-00}
\end{aligned}
$$

\section{Results and Discussion}

The data points in the aerodynamic database are presented here. Figures 6 and 7 are formatted to easily pick data from the plots. This formatting includes sliding vertical axes where necessary to keep lines from overlapping, and plotting data versus Mach number and Knudsen number rather than angle-of-attack. Trends described in the following sections may not be as clearly evident due to this formatting.

For all aerodynamic coefficients, the reference length is the maximum diameter of the capsule, $D=$ $2.648 \mathrm{~m}$. The reference area, $\mathrm{S}$ is projected area at the maximum diameter, $S=5.505 \mathrm{~m}^{2}$. The moment reference point for each pitching moment figure is provided in the figure. The center-of-gravity $(\mathrm{cg})$ position $\left(x_{c g}\right)$ for the MER entry capsules was 0.26 body diameters aft of the nose. All data is plotted in terms of total angle-of-attack. This is valid for axisymmetric vehicles such as MER. For use in 6-DoF simulations, the MER aerodynamic database decomposes this total angle-of-attack data into full 6-DoF coefficients described in Figure 1 for a given angle-of-attack and sideslip.

\section{A. Rarefied Flow: Free molecular and transitional regimes}

Plotted in Figures 6a-c are the static aerodynamic force and moment coefficients versus Knudsen number across the transitional flight regime. The symbols mark specific points calculated by DAC, DACFREE or LAURA. The curves show the interpolation between these points as performed in the aerodynamic database subroutine.

The pitching moment coefficient data in Figure 6a show how the MER capsule changes from being statically unstable $\left(C_{m}>0\right)$ in the free molecular regime to statically stable $\left(C_{m}<0\right)$ in the continuum 


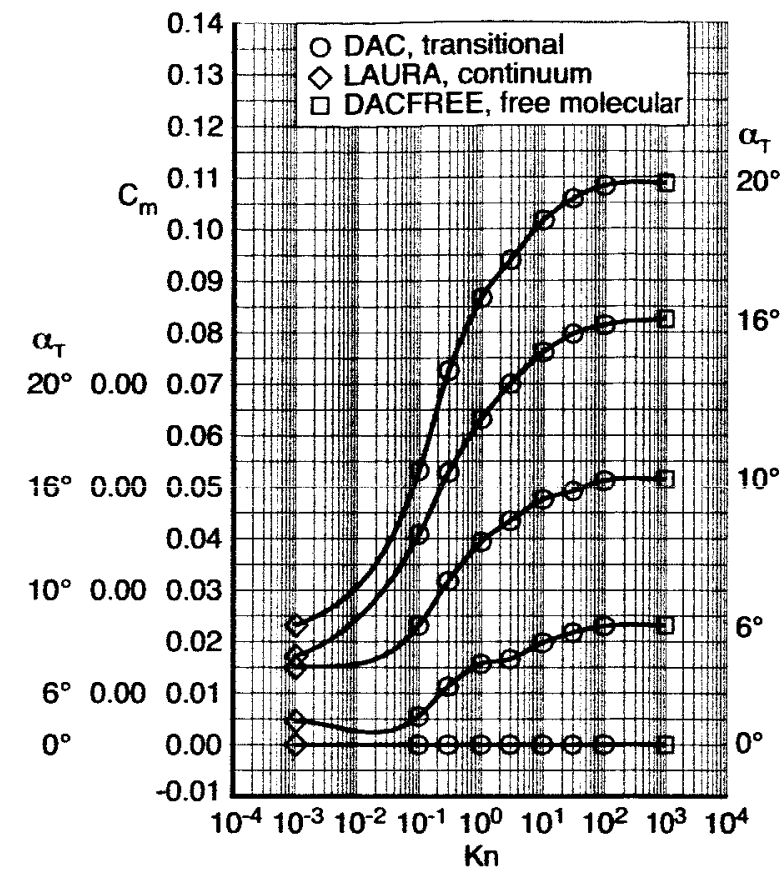

(a) Pitching moment coefficient

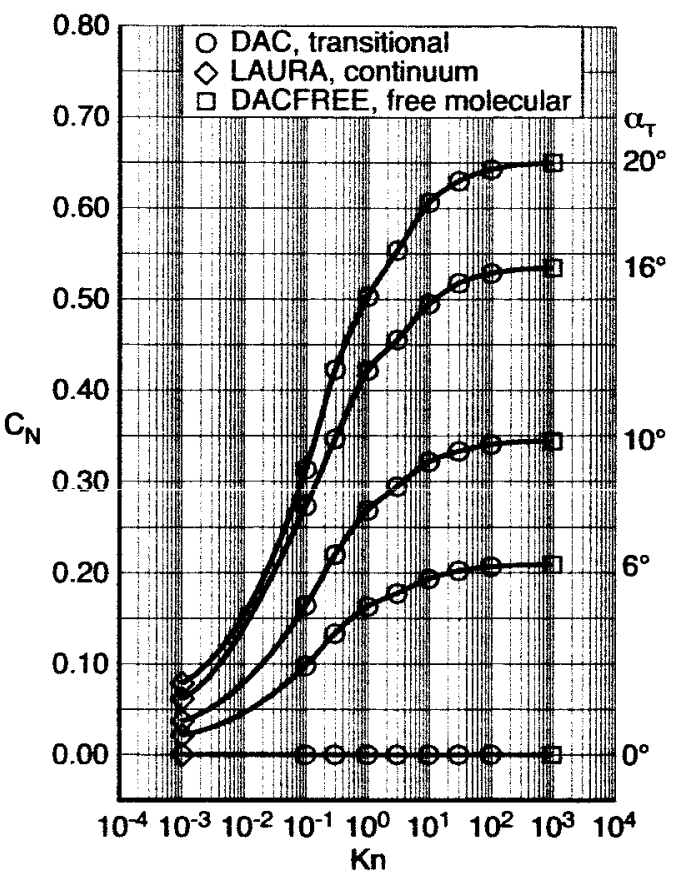

(b) Normal force coefficient

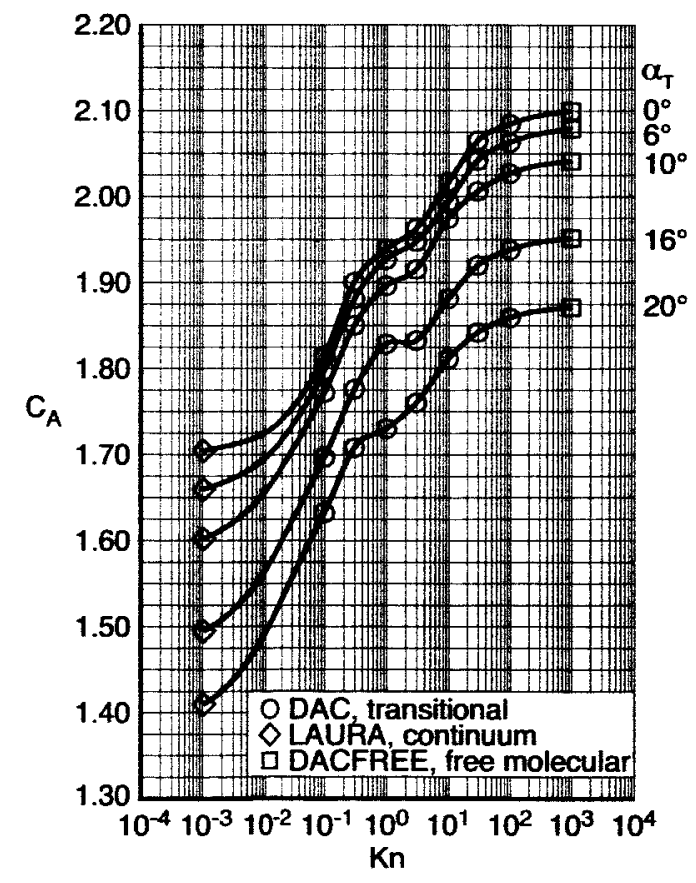

(c) Axial force coeffficient

Figure 6. Transitional regime static aerodynamics (moment reference point $=0.2498$ diameters aft of nose) 
regime. This switch occurs at a Knudsen number of approximately 0.25 , where the capsule has almost no pitching moment at any angle-of-attack. This portion of the transitional regime is characterized by the changing influences of the shear and normal forces on the capsule. This change in relative contribution drives the change in stability. Note that there is a somewhat large gap between the LAURA-based data at the low Knudsen number end and the first DAC solutions. This region is of such high particle density that current computational capabilities can not yet adequately model enough molecules to get accurate DSMC solutions. Yet this density is still low enough that LAURA can not accurately model the flow either. The interpolation routine used in the database blends the data in this range smoothly. Assuming there are no anomalous phenomena in this Knudsen number range (a reasonable assumption), this bridging should closely approximate the actual behavior of the MER capsule in this region. To be conservative, larger uncertainties are applied to this region to account for any unexpected phenomena.

The normal force and pitching moment coefficients are shown in Figure $6 \mathrm{~b}$ an c. The curves are fairly smooth and show the transition from the free molecular $C_{N}$ and $C_{A}$ values to the continuum values. Again, additional uncertainties are added for conservativism to account for any possible anomalous behavior which current computational capabilities are unable to resolve between the continuum regime and first acceptably resolved DSMC solutions.

After Mars Pathfinder successfully landed, Moss et $a^{12}$ performed extensive DSMC analysis of the rarefied flow regime for that mission. The principle difference between the MPF and MER calculations is the entry velocity. Matching Knudsen number and velocity almost exactly duplicates the MPF data. Comparisons indicate that the different wall temperature boundary conditions and gas composition differences had minimal impact on the aerodynamics.

\section{B. High Speed Continuum: Hypersonic and supersonic flight regimes}

Figures $7 \mathrm{a}-\mathrm{c}$ show the variation of the aerodynamic forces and pitching moment with Mach number for several total angles-of-attack up to $16^{\circ}$. The symbols again show individual data points in the database, while the lines show the interpolation performed in the database subroutine. Diamond symbols indicate cases where the base pressure correction (described previously) is applied.

The pitching moment curves (Figure 7a) show typical stability trends for a $70^{\circ}$ sphere-cone with some features unique to the MER capsule/trajectory combination. There is a region of bounded instability at Mach 16. This instability can be seen in Figure 7a where the $\alpha=2^{\circ}$ curve is positive. The Mach 16 instability occurs because of a change in the forebody pressure distribution caused by the gas chemistry of the forebody flow changing from equilibrium to perfect gas. This transition correlates with a shift in the lee-side sonic line from the shoulder to the nose cap. At Mach 27 there is another bounded instability for the MER flight vehicles' center-of-gravity position $\left(x_{c g} / D=0.26\right)$. The MER capsule is unstable in this region due to the gas chemistry changing from frozen flow to equilibrium and also correlates with the sonic line transitioning to the nose cap. Similar instabilities were predicted for the MPF entry and verified by post-flight reconstruction. However, the locations of these instabilities are trajectory specific. Had the MPF database been used for MER, the instability at Mach 27 would not have been identified in the entry predictions. A description of the flow physics which drives these instabilities as well as post-flight verification of the Mars Pathfinder aerodynamics are presented by Gnoffo et al. ${ }^{13}$

Figure $7 \mathrm{~b}$ shows the variation of the normal force coefficient with Mach number for the angles-of-attack of the database. The normal force coefficient is small at low angles-of-attack for blunt vehicles like the MER entry capsule, and these curves are in agreement with vehicles like MPF and Viking.

Figure $7 \mathrm{c}$ shows the axial force coefficients versus Mach number. Note that the $C_{A}$ axis for each angle-ofattack curve is labeled individually. The $C_{A}$ increments between each grid-line are the same for each curve. The overall trends in this data are as expected and in keeping with the behavior seen by Mars Pathfinder and Viking. At the higher Mach numbers $(M=16-26)$, the axial force coefficients are fairly invariant with Mach number. For lower Mach numbers, there is a gradual decrease in the axial force coefficient as the flow physics begin to transition to the transonic regime. The higher angle-of-attack cases show a slight increase in $C_{A}$ below Mach 5. This appears to be related to changes in the relative contributions to $C_{A}$ from the forebody and backshell. The Mach $=1.5$ data points show that $C_{A}$ is again decreasing as the pressure on the forebody rapidly decreases. These features are in qualitative agreement with the Mars Viking database. ${ }^{6}$ This comparison will now be discussed. 


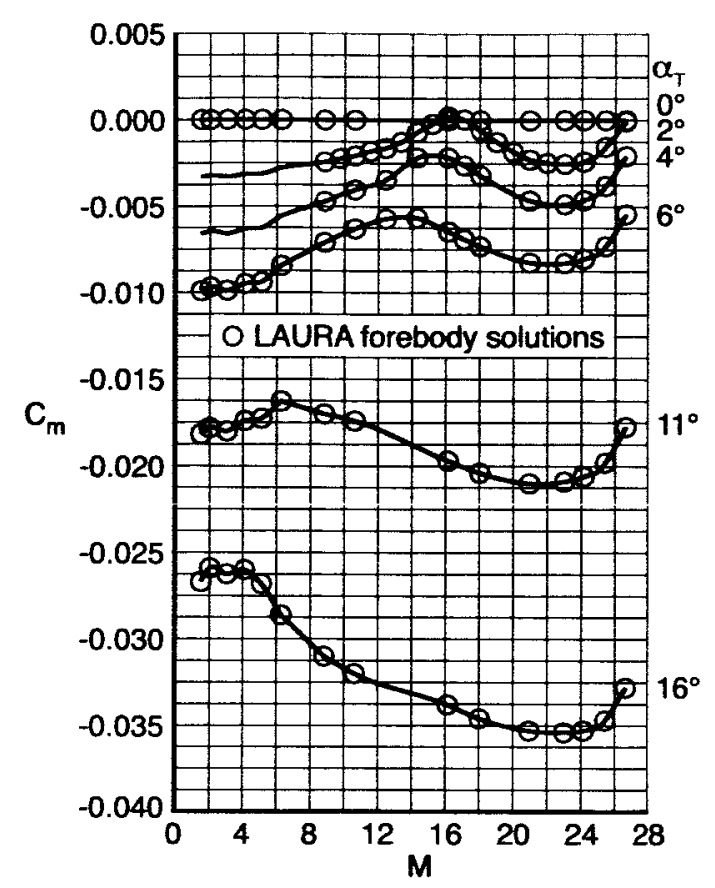

(a) Pitching moment coefficient

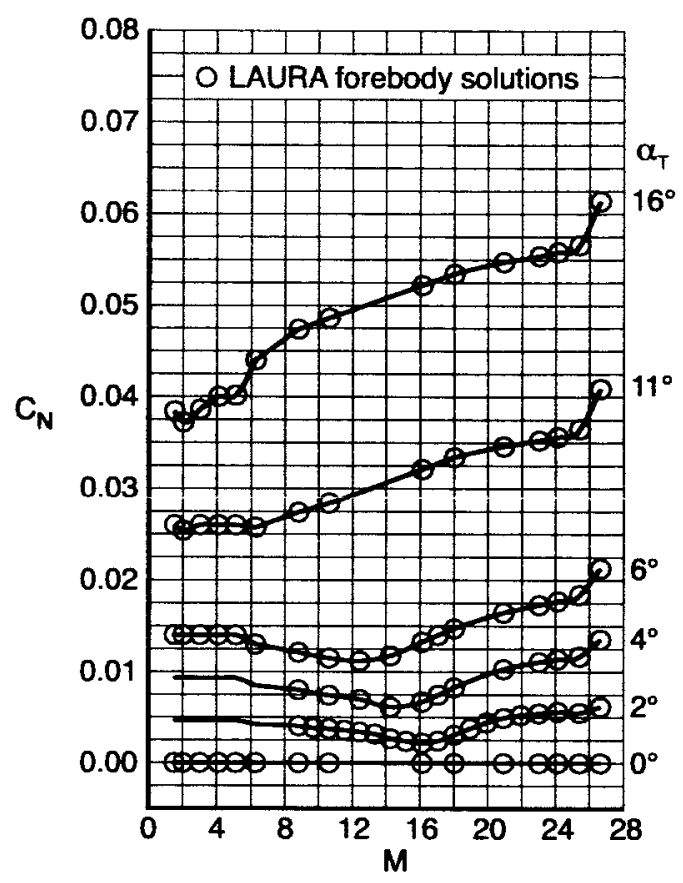

(b) Normal force coefficient

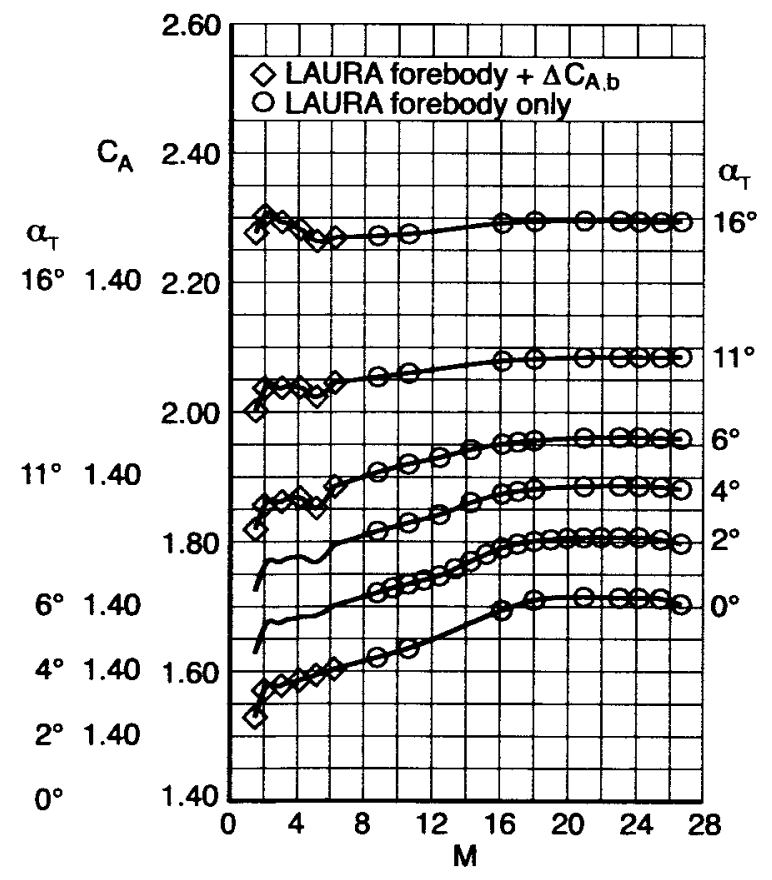

(c) Axial force coefficient

Figure 7. Continuum regime static aerodynamics (moment reference point $=0.2498$ diameters aft of nose) 


\section{Axial force coefficient comparison with heritage data}

Figure 8 shows a comparison of the MER database below Mach 7 against several heritage data sources. The forebody contribution for both MER and MPF are shown. The differences between these two curves are trajectory related and account for the differences in the total $C_{A}$ curves. The addition of a base correction to the MER forebody CFD data results in an axial force curve very similar in form to the Viking wind tunnel ${ }^{6}$ and ballistic range data. ${ }^{7}$ The full LAURA solutions show a trend which diverges from all available heritage data. Based on this comparison, it was decided to adopt the MPF approach. Along with agreeing with heritage data, the base corrected data was more conservative from an entry timeline perspective than the full LAURA solutions. Note that the MER ballistic range data is actually more conservative still. However, determining the pitch damping was the primary objective of the MER ballistic range test program and the resolution of $C_{A}$ has known caveats (very few data points at low Mach and low angles). ${ }^{8}$ As a result, the variation of the static coefficients with Mach number and angle-of-attack were not sufficiently resolved to incorporate the ballistic range values within the flight database.

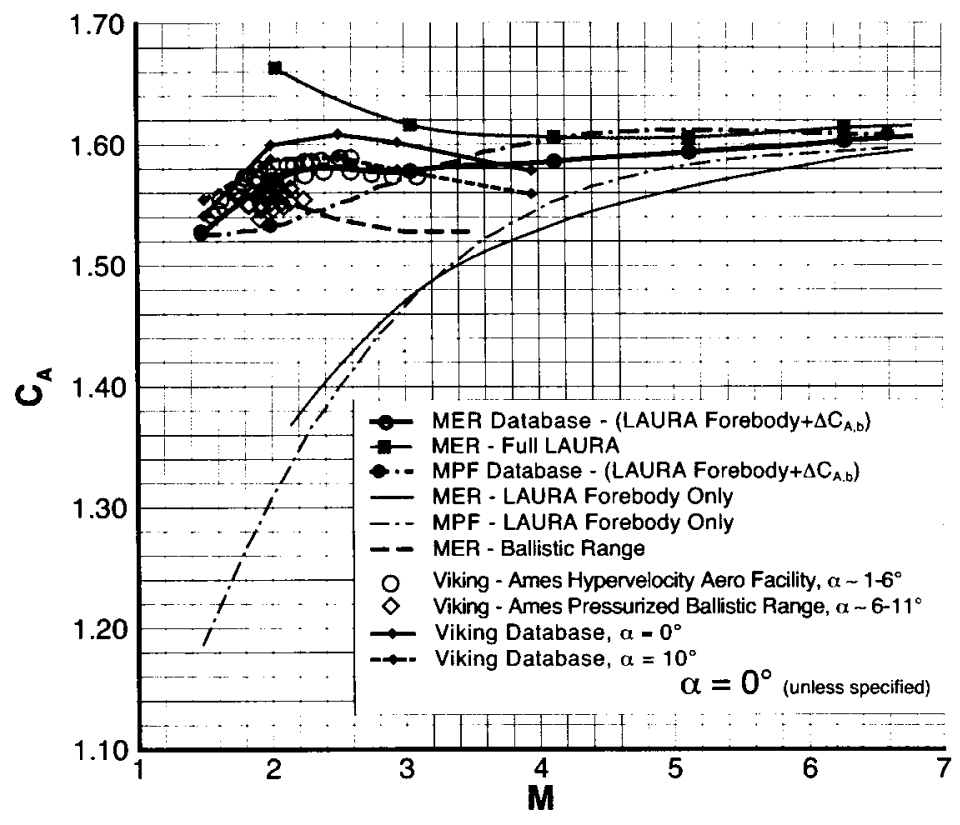

Figure 8. Comparison of heritage axial force coefficient data with MER database

\section{Bounded Instabilities}

In Figure 7a, two bounded instabilities are evident where the aeroshell is unstable at small angles-of-attack. These instabilities were first predicted by Gnoffo $^{13}$ for the Mars Pathfinder mission and confirmed by flight data. However, for the MER mission it was unknown if the first instability would be encountered as the MER entry velocity is slower than the velocity at which that instability occurred during the MPF entry. Figure 9 shows $C_{m}$ curves at $\alpha=2^{\circ}$ and $5^{\circ}$ for the MER and MPF databases. The two MPF bounded instabilities are clearly seen. LAURA CFD does predict that both instabilities would be encountered during the MER entries. However, due to the slower entry velocity, both instabilities are less severe. As Gnoffo described for MPF and LAURA CFD showed, the two instabilities both correlate with a transition of the sonic line from the shoulder to the nose cap on the lee side of the forebody resulting in high pressures out near the lee-side shoulder. The first instability (higher velocity) results from the gas chemistry behind the bow shock changing from frozen flow to equilibrium. The second instability (lower velocity) occurs as the flow behind the bow shock changes from equilibrium to perfect gas chemistry. The lower energy entry results in less dramatic changes in pressure distribution with changes in gas chemistry. 
The highest velocity point in Figure 9 corresponds to the first continuum point along the entry trajectory. The velocity is essentially constant prior to that point and the static stability is driven by the freestream conditions only (density/Knudsen number). Including the aerodynamics for the transitional regime, the MER database predicts that the capsule would experience shallow stability (and two bounded instability regions at low angles-of-attack less severe than MPF) from entry interface until approximately Mach=14. The capsule was predicted to encounter less severe bounded instabilities (lower trim angles-of-attack) than MPF experienced, but it would not be as statically stable while decelerating at velocities between the two instabilities. The differences illustrated here are due entirely to differences in the entry trajectories. These changes in the predicted aerodynamic performance of MER indicates the necessity of a new database. Had the Pathfinder database been used "as-is" for MER, the first instability and the overall less stable performance of the MER capsule would not have been predicted.

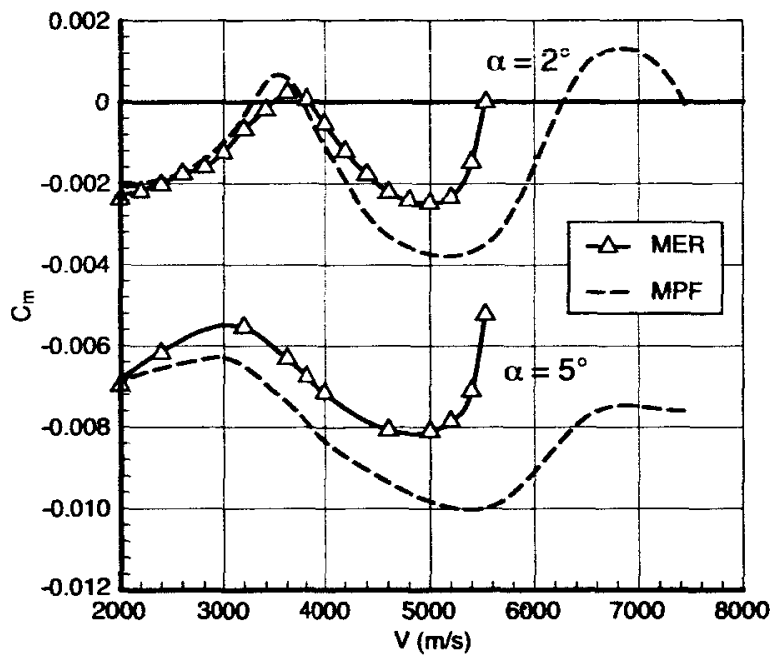

Figure 9. Bounded instabilities for MER and MPF (Moment reference point $=0.26$ diameters aft of nose)

\section{Conclusions}

The Mars Exploration Rover aerodynamic database has been developed based on Viking and Mars Pathfinder heritage. Analysis has shown that the computational advances developed for Mars Pathfinder are directly applicable to MER and recent advances in computational efficiency have resulted in the highest fidelity database for a Mars entry to date. Computational capabilities have improved in recent years, making DSMC and CFD solutions more affordable. As a result, the MER database was able to incorporate DSMC data points across the transitional regime. Also, the hypersonic regime was populated entirely with LAURA solutions and the supersonic regime had far more LAURA solutions than MPF. The MER database is much improved over MPF.

While the outer mold lines of the MER and MPF capsules were of very similar design, differences in their entry trajectories required a new database to accurately describe the flight characteristics of MER in the hypersonic flight regime. Analysis of the MER trajectory shows differences in the bounded instabilities that would not have been predicted using the MPF database.

Future work will compare the flight telemetry from the two successful MER entries, extracting static aerodynamics with parameter identification techniques. While successfully used for MPF and MER, improving on the empirical base pressure correction used for the supersonic axial force values should also be explored in the future (i.e. replacing the correction with improved computational solutions). This would improve future mission simulations and increase the fidelity of atmosphere reconstruction already performed for MPF and MER. 


\section{References}

${ }^{1}$ Lebeau, G. J., "A How To Guide For The DAC Series," NASA JSC, Revision 95v2, September 1997.

${ }^{2}$ Cheatwood, F. M. and Gnoffo, P. A., "User's Manual for the Langley Aerothermodynamic Upwind Relaxation Algorithm (LAURA)," NASA TM 4674, 1996.

${ }^{3}$ Brauer, G. L., Cornick, D. E., and Stevenson, R., "Capabilities and Applications of the Program to Optimize Simulated Trajectories (POST)," NASA CR 2770, February 1977.

${ }^{4}$ Tobak, M. and Wehrend, W. R., "Stability Derivatives for Cones at Supersonic Speeds," NACA TN-6788, NACA, September 1956. 1994.

${ }^{5}$ Bird, G., Molecular Gas Dynamics and the Direct Simulation of Gas Flows, Oxford University Press Inc., New York,

${ }^{6}$ Flaherty, T. M., "Aerodynamics Data Book," TR- 3709014, Martin Marietta Corporation, 1972.

${ }^{7}$ Sammonds, R. I. and Kruse, R. L., "Viking Entry Vehicle Aerodynamics at $M=2$ in Air and Some Preliminary Test Data for Flight in CO2 at $M=11$," Tech. Rep. NASA TN D-7974, 1975.

${ }^{8}$ Schoenenberger, M., Hathaway, W., Yates, L., and Desai, P., "Ballistic Range Testing of the Mars Exploration Rover Entry Capsule," AIAA 2005-0055, January 2005.

${ }^{9}$ Williams, S. D. and Curry, D. M., "Thermal Protections Materials - Thermophysical Property Data," NASA Ref. Publication 1289, December 1992.

${ }^{10}$ Riley, C. and Cheatwood, F., "Distributed-Memory Computing with the Langley Aerothermodynamic Upwind Relaxation Algorithm (LAURA)," Advances in Engineering Software, Vol. 29, No. 3-6, July 1998, pp. 317-324.

${ }^{11}$ Desai, P. N., Schoenenberger, M., and Cheatwood, F. M., "Mars Exploration Rover Six-Degree-of-Freedom Entry Trajectory Analysis," AAS 03-642, August 2003.

${ }^{12}$ Moss, J. N., Blanchard, R. C., Wilmoth, R. G., and Braun, R. D., "Mars Pathfinder Rarefied Aerodynamics: Computations and Measurements," Journal of Spacecraft and Rockets, Vol. 36, No. 3, pp. 330-339.

${ }^{13}$ Gnoffo, P. A., Braun, R. D., Weilmuenster, J. K., Mitcheltree, R. A., Engelund, W. C., and Powell, R. W., "Prediction and Validation of Mars Pathfinder Hypersonic Aerodynamic Database," Journal of Spacecraft and Rockets, Vol. 36, No. 3, May-June 1999, pp. 367-373. 